\title{
Effect of Anodal tDCS on Articulatory Accuracy, Word Production, and Syllable Repetition in Subjects with Aphasia: A Crossover, Double-Blinded, Sham- Controlled Trial
}

\author{
Camila Vila-Nova • Pedro H. Lucena · Rita Lucena - Giulia Armani-Franceschi • \\ Fernanda Q. Campbell
}

Received: November 1, 2018 / Published online: August 20, 2019

(c) The Author(s) 2019

\section{ABSTRACT}

Introduction: Transcranial direct-current stimulation (tDCS) has been used to modulate and induce changes in brain function and excitability. tDCS is a promising tool for the treatment of aphasia.

Enhanced Digital Features To view enhanced digital features for this article, go to https://doi.org/10.6084/ m9.figshare.9169760.

Equally contributing authors: Camila Vila-Nova and Pedro H. Lucena.

C. Vila-Nova $(\bowtie)$

União Metropolitana de Educação e Cultura-

UNIME, Kroton and University of the State of Bahia, Avenida Luis Tarquínio Pontes, 600 Lauro de Freitas, BA, Brazil

e-mail: camivnova@gmail.com

\section{P. H. Lucena}

Bahiana School of Medicine and Public Health, Av. Dom João VI, 275, Brotas, Salvador, BA, Brazil

\section{R. Lucena}

School of Medicine of Bahia, Federal University of Bahia, Av. Luis Viana Filho S/N, Canela, Salvador, BA, Brazil

G. Armani-Franceschi

Federal University of Bahia, Av. Luís Viana Filho

S/N, Canela, Salvador, BA, Brazil

F. Q. Campbell

The Heller School for Social Policy and

Management, Brandeis University, 415 South Street,

Waltham, MA, USA
Objective: To evaluate whether tDCS improves articulatory accuracy and speech production in patients with aphasia after stroke.

Methods and Results: Twelve right-handed subjects participated in a double-blind, shamcontrolled, crossover offline trial. We assessed (1) articulatory accuracy at a naming task, (2) number of words correctly produced, (3) number of syllables repeated correctly, and (4) qualitative assessment of speech. Articulatory accuracy improved when using tDCS over Broca's area in subjects with aphasia post-stroke $(p \leq 0.05)$. Qualitative improvement in the naming and syllable repetition tasks was observed, but the difference was not statistically significant (respectively, $p=0.15$ and $p=0.79$ ). Conclusion: The current results corroborate the potential of tDCS to be used as an alternative and complementary treatment for individuals with aphasia.

Keywords: Aphasia; Broca's area; Language rehabilitation; Speech accuracy; Stroke; Transcranial direct-current stimulation (tDCS)

\section{INTRODUCTION}

Aphasia is one of the most devastating poststroke sequelae, affecting nearly $30 \%$ of individuals that experienced an ischemic stroke [1]. Partial language recovery is common in these subjects and tends to occur within the first 
months after the event. Nearly $40 \%$ of individuals with severe aphasia present considerable improvement during the first 3 months after the stroke [2]. Nonetheless, language deficit persists and speech-language therapy is necessary to avoid a decline of cognitive functions [3].

The process of word articulation involves the motor cortex, primarily the Broca's area and the anterior insula, as well as areas located at the primary motor cortex on both hemispheres [4]. Aphasia usually causes linguistic processing deficits, impairment of motor and articulatory speech ability [5], often impairing verbal comprehension.

Rehabilitative techniques have been developed to help a patient recover or to maximize language function after neurologic damage. Although speech-language therapy is the gold standard for rehabilitation of individuals with post-stroke aphasia, the results usually plateau or are limited [6]. Recent studies, however, suggest that transcranial direct-current stimulation (tDCS) can improve language by eliciting changes in cortical excitability and help with post-stroke rehabilitation of patients [7].

tDCS is a non-invasive technique able to change neuronal excitability of the cortex. The resulting polarity can be either excitatory (anodal current) or inhibitory (cathodic current). The polarity is generated by an electrical circuit formed by the positioning of different-sized electrodes, placed in different montages to stimulate the cortex. This procedure has been tested as a tool to enhance different cognitive and motor functions in both healthy subjects and patients with a variety of conditions $[8,9]$.

The number of studies using tDCS to improve language ability in individuals with post-stroke aphasia is rising. These studies vary in terms of polarity used (anodal vs. cathodal) and brain region stimulated. The choice of area to be stimulated is determined by the current knowledge of language neuroanatomy. The Brodmann area 44 on the left hemisphere (which comprises Broca's area) is considered the main area for speech motor programming $[10,11]$. Researchers usually choose this area for stimulation during tDCS in order to improve language processing [12].
Anodal current has been applied in different perisylvian areas at the language-dominant hemisphere (Broca, temporoparietal, temporoparietoociptal), as well as in perilesional areas [6, 12-16]. The cathodal current has been applied in correspondent areas on the nondominant hemisphere [17-19] or in areas deemed important for the naming process, such as the temporoparietal cortex [18, 20]. Most studies demonstrate that anodal stimulation on the left frontal cortex produces improvement in the performance of naming tasks $[6,12-16,18,20]$. To the best of our knowledge, there are few studies reporting positive results using cathodal tDCS (C-tDCS) in homologue areas $[15,18]$. Additionally, Iyer et al. described decrease in verbal fluency after using C-tDCS in the left prefrontal cortex [21]. These findings suggest that anodal tDCS (A-tDCS) may be the best option for aphasia treatment in terms of polarity. Nonetheless, a recent meta-analysis highlighted the lack of enough evidence supporting the efficacy of this technique on the treatment of aphasia and called for more randomized clinical trials in this area [6].

Parameters used across studies are heterogeneous, and it is not yet clear which areas should be stimulated and what intensity and electrode size should be used. De Aguiar et al. suggest some parameters to be used on subjects with aphasia [15], but the best timing for intervention, brain region to stimulate, and the duration of the session are yet to be determined [6]. Besides the variation in parameters during stimulation, studies also diverge in terms of outcomes. Researchers assessing the impact of tDCS on aphasia usually consider changes in verbal behaviors, using naming and fluency tests, as well as naming reaction time $[12,16]$. Most studies regarding the effects of tDCS on language in patients with aphasia evaluate the degree of improvement in naming ability $[12,14,17,22]$. Motor aspects of speech are not commonly studied when Broca's area is stimulated using tDCS. Articulatory accuracy, however, is generally compromised in aphasia and warrants additional study. Speech accuracy was included as a measure in order to fill this gap in the neuromodulation literature. 
Table 1 Demographic and clinical data of 12 adults with aphasia post-stroke at study baseline

\begin{tabular}{|c|c|c|c|c|c|c|c|c|c|}
\hline \multirow[t]{2}{*}{ Subject } & \multicolumn{9}{|c|}{ Characteristics } \\
\hline & Gender & $\begin{array}{l}\text { Age } \\
\text { (years) }\end{array}$ & $\begin{array}{l}\text { Number } \\
\text { of strokes }\end{array}$ & $\begin{array}{l}\text { Stroke } \\
\text { type }\end{array}$ & $\begin{array}{l}\text { Stroke } \\
\text { time } \\
\text { (months) }\end{array}$ & $\begin{array}{l}\text { Aphasia } \\
\text { type }\end{array}$ & $\begin{array}{l}\text { Arterial } \\
\text { hypertension }\end{array}$ & Smoking & $\begin{array}{l}\text { Alcohol } \\
\text { consumption }\end{array}$ \\
\hline 1 & $\mathrm{~F}$ & 53 & 1 & I & 40 & Transcortical & Yes & No & No \\
\hline 2 & $\mathrm{~F}$ & 80 & 1 & I & 55 & Transcortical & Yes & Yes & No \\
\hline 3 & M & 46 & 1 & $\mathrm{H}$ & 42 & Broca's & Yes & Yes & No \\
\hline 4 & M & 59 & 1 & $\mathrm{H}$ & 50 & Broca's & Yes & No & No \\
\hline 5 & M & 58 & 1 & I & 15 & Anomic & Yes & Yes & Yes \\
\hline 6 & M & 55 & 1 & $\mathrm{H}$ & 31 & Broca's & Yes & Yes & No \\
\hline 7 & $\mathrm{M}$ & 65 & 2 & I & 34 & Anomic & Yes & No & Yes \\
\hline 8 & $\mathrm{M}$ & 69 & 1 & I & 19 & Anomic & Yes & Yes & Yes \\
\hline 9 & $\mathrm{~F}$ & 33 & 1 & I & 46 & Broca's & Yes & No & No \\
\hline 10 & $\mathrm{~F}$ & 67 & 4 & I & 16 & Anomic & Yes & No & Yes \\
\hline 11 & $\mathrm{~F}$ & 63 & 5 & I & 70 & Conduction & Yes & No & No \\
\hline 12 & $\mathrm{~F}$ & 43 & 1 & I & 26 & Broca's & No & No & No \\
\hline
\end{tabular}

$M$ male, $F$ female, $I$ ischemic, $H$ hemorrhagic, $A H$ arterial hypertension

Thus, as aphasia rehabilitation techniques are still limited and as tDCS is considered a promising therapeutic tool, we assessed the effect of A-tDCS on naming ability and on the speech motor system in Brazilian patients with aphasia after stroke. We hypothesized that A-tDCS would improve articulatory accuracy, and increase the number of words produced, and the number of syllables repeated correctly at a naming task. We also examined qualitative perception of change after treatment.

\section{METHODS}

\section{Subjects}

Twelve right-handed, native Portuguese speakers with chronic post-stroke aphasia (6 male and 6 female, aged $57.6 \pm 12.7$ years) were included in the study. These patients were recruited in treatment centers of a capital at the northeast region of Brazil. Single stroke occurred in 75\% of the sample. All subjects presented right hemiparesis. Time after onset of stroke varied from 15 to 70 months ( $37 \pm 16.8$ months). All individuals, except one, presented arterial hypertension (91.6\%). Before the stroke, $41.6 \%$ usually consumed alcohol (3 to 5 times per week) and $33.3 \%$ were smokers. None presented cardiac disease. Table 1 shows the characteristics of all participants.

Participants had never received tDCS. Subjects were first evaluated by a neurologist to define the stroke diagnosis using the parameters defined by the Trial of Org 10172 in Acute Stroke Treatment (TOAST) [23]. All had a lesion that occurred at least a year prior to the study and all had been receiving traditional speechlanguage therapy once a week for at least 6 months. The study excluded patients with moderate to severe cognitive deficit (according to DSM-IV criteria), receptive language deficit, auditory loss, cardiac disease, pacemaker use, 
seizures within the past 6 months, and orofacial malformation or malocclusion that could compromise speech praxia. Additional exclusion criteria applied after the beginning of the study included changes in pharmacological and nonpharmacological treatments, infection, head trauma, another stroke episode, and distress during tDCS sessions.

In order to classify the type of aphasia, a speech-language pathologist not involved in the experiment assessed all participants. The therapist used a Brazilian ecological test for classification of aphasia, the "Teste de Reabilitação das Afasias: Rio de Janeiro" [24]. This test includes picture naming, verbal and non-verbal apraxia, performance on repetition of words and phrases, reading, and spontaneous speech and writing ability. After receiving the aphasia classification, participants with speech articulatory deficits were included. As previous studies have presented results for heterogeneous samples, and due to the difficulty of recruitment for tDCS studies, we decide to include all types of expressive aphasia. Subjects received medical approval for participation and gave their written consent. All procedures performed in this study were in accordance with the ethical standards of the institutional and/or national research committee and with the 1964 Helsinki Declaration and its later amendments. The study was approved by the institutional review

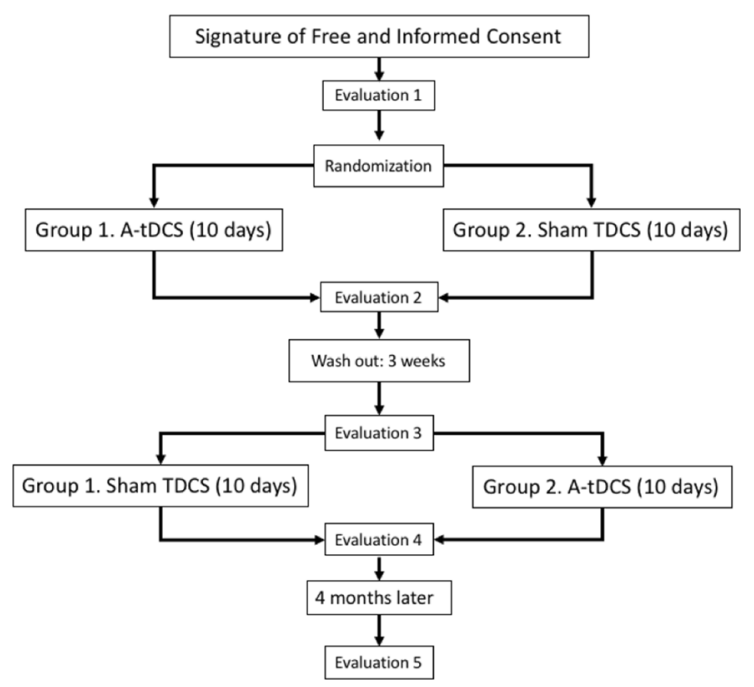

Fig. 1 Study design. Outline of study procedures timeline board (IRB) of Hospital Santo Antonio, Salvador-Bahia, Brazil (\#22/07).

\section{Study Design}

This was a crossover, double-blinded, shamcontrolled offline study. Figure 1 depicts the experimental design. Participants were randomly assigned to two groups with different orders of stimulation (sham followed by active tDCS or vice versa). Follow-up assessment was performed at the end of each intervention period. A qualitative follow-up occurred at 1 week and at 4 months after the end of the intervention.

tDCS was delivered by a battery-driven, constant-current stimulator through a pair of saline-soaked sponge electrodes $(7 \mathrm{~cm} \times 5 \mathrm{~cm})$ which were kept in place by elastic bands. We used a tDCS device developed at Mackenzie University-Sao Paulo-SP, Brazil [25]. As the majority of previous studies have shown positive effects of anodal tDCS (A-tDCS), we chose this polarity $[6,12-15,18,20,26]$. The anodal electrode was placed on the left side of patient's head (Broca's area) following the 10/20 EEG system [27]. Broca's area was determined by the intersection of the lines formed by points Cz-F7 and Fz-T3. These points, previously used in other studies [22, 25] were identified using a measuring tape. The cathodic electrode was placed at the right supraorbital area.

The stimulation lasted $20 \mathrm{~min}$ using a current of $1 \mathrm{~mA}$ over the course of 10 days (5 days during a week, weekend interval, and another 5 days) $[12,28]$. The duration and intensity level were chosen based on previous studies that assessed safety of tDCS in humans [18, 29]. At the onset of both active and sham stimulation, the current was increased in a ramp-like fashion eliciting a transient tingling sensation on the scalp that faded over $5 \mathrm{~s}[28,30]$. The researchers who conducted the assessments were blind to the study design. Since the goal was solely to investigate the effect of A-tDCS, an offline design (task and tDCS are not performed concurrently) was adopted [31].

After the washout period, patients treated with anodal stimulation received sham 
treatment and vice versa. Given the lack of a standard protocol, we defined the washout period based on the findings of previous studies. $[12,18]$. This interval varied from $24 \mathrm{~h}$ to 3 weeks $[13,17]$. To avoid carryover effects, we adopted a conservative washout period of 3 weeks.

\section{Trial Registration}

The trial was not registered because at the time the study was conducted, registration of clinical trials in Brazil was still not a consolidated practice. Reasonable effort has been made since to apply for retrospective trial registration, but has not been possible.

\section{Instruments and Measure}

To assess the effects of A-tDCS, subjects in the two groups (anodal and sham) were evaluated before and after the 10-day intervention using the study naming and repetition tasks. Preassessment occurred at the same day participants received the first tDCS session, for both sham and anodal groups. Post-assessment occurred at the last day of intervention, 15-20 min after the session.

\section{Naming task}

The picture-naming task of the Snodgrass test was used $[32,33]$. The same 20 pictures were used for both sham and anodal groups, before and after the 10-day intervention period. These pictures were uploaded on Chronos software and were selected based on characteristics such as word length, visual complexity, and phonologic information (e.g. syllable articulatory complexity). Picture-naming tasks are widely used in tDCS studies to test individuals without [34] and with [10, 12] aphasia. A speech sample was recorded using the software Sony Sound Forge Pro 9.0. We collected these samples using a Stile VG Felitron voice tube headset, connected to a notebook with a Windows 2007 operational system.

Two measures related to the naming task were created: (1) number of words produced correctly, and (2) articulatory accuracy. Two blinded speech-language pathologists (SLPs) who received training in order to standardize the assessment and increase inter-rater reliability conducted the assessment of speech production and the overall speech praxis. Using the list of 20 words supplied in the task, they compared each word's production before and after the 10-day stimulation period and rated the subsequent production as being "the same", "better accuracy", and "less accuracy". The SLPs recorded the total number of words that presented "better accuracy" for each study participant (articulatory accuracy). The criteria used to judge accuracy was articulatory precision to the extent that the evaluator was able to understand the target word. Although articulatory accuracy is a subjective parameter, it allows judgment based on real-world experiences of subjects with aphasia. The measure translates how well a listener understands target words. This has a pragmatic and social value because we are observing language from a functional and interactional perspective $[35,36]$.

\section{Repetition Test: Syllables}

The repetition task consisted of 150 syllables created from the combination of usual consonant and vowel sounds of Brazilian Portuguese: consonant + vowel and consonant + vowel + consonant (list available from the authors). This task was used to assess isolated production of phonemes. The variable of interest was the number of syllables repeated correctly. Judging syllable production reduces the influence of lexical, syntactic, and semantic levels on phonemic production. The goal was to assess oral production-articulatory ability without the influence of linguistic processing, which happens during naming tasks. The measure was the number of correctly produced syllables. We avoided including syllables that could have semantic value, such as the syllable "pe", which is similar to the monosyllabic word "pé" ("foot" in Portuguese) or the syllable "mar", because it is equal to the monosyllabic word "mar" ("sea", in Portuguese). Syllable production was compared before and after the 10-day stimulation period and rated as "the same", "better accuracy", and "less accurate". 
Qualitative Assessment: Individual Outcomes

At 1 week and 4 months after the end of the study (follow-ups 1 and 2), the participants, a family member, and the participant's SLP were asked about changes in the participant's speech. A researcher not involved with study procedures asked participants and family members: "Have you noticed any changes in your (the participant's) speech after the treatment?" The same researcher asked therapists: "Have you noticed changes in the participant's word production and speech quality?"

\section{Statistical Analysis}

The non-parametric Mann-Whitney test for paired samples was used to compare pre- and post-stimulation scores on the naming test (number of words produced correctly and articulatory accuracy) and the syllable repetition task (number of syllables produced correctly). To calculate these scores, the difference between pre- and post-intervention for each subject in the intervention group was computed. This difference was then divided by the number of correct productions pre-intervention and averaged for each group. The Wilcoxon paired test was used to compare the average number of words produced with better accuracy at each treatment (sham and active A-tDCS). Cohen's kappa was used to assess inter-rater agreement for the two referees. The same index was computed for inter-rater agreement at the

Table 2 Pre- and post-stimulation mean differences for patient's performance on word production and syllable repetition

\begin{tabular}{llll}
\hline Variable & $\begin{array}{l}\text { Sham } \\
\text { A-tDCS } \\
\text { mean } \\
(\text { SD })\end{array}$ & $\begin{array}{l}\text { Active } \\
\text { A-tDCS } \\
\text { mean } \\
(\text { SD })\end{array}$ & P value* \\
\hline $\begin{array}{l}\text { Mean difference in } \\
\text { correct words }\end{array}$ & $0.24(0.58)$ & $0.01(0.37)$ & 0.1540 \\
$\begin{array}{l}\text { Mean difference in } \\
\text { correct syllables }\end{array}$ & $0.08(0.18)$ & $0.19(0.50)$ & 0.7896 \\
\hline $\begin{array}{l}\text { SD standard deviation } \\
* \text { Wilcoxon test for paired data }\end{array}$ & & \\
\hline
\end{tabular}

qualitative assessment. For the qualitative assessment, we considered five categories, according to kappa values: no agreement $(<0)$, slight agreement (0-0.19), fair agreement (0.2-0.39), moderate agreement (0.4-0.59), substantial agreement (0.6-0.79), and almost perfect agreement (0.80-1) [37]. The software $R$ version 2.11.1 [38] was used to perform the Mann-Whitney matched pairs test, and SPSS was used for all other analyses. Differences that achieved a $p$ value $\leq 0.05$ were considered statistically significant. We used the Bonferroni method to correct for multiple comparisons.

\section{RESULTS}

\section{Word Production and Syllable Repetition}

As shown in Table 2, non-parametric tests suggest that there was no statistical difference in the number of words $(p=0.1540)$ and syllables produced correctly $(p=0.7896)$ after the treatment.

\section{Articulatory Accuracy Analysis}

The Wilcoxon paired test was used to compare the average number of words produced with better accuracy at each treatment (sham and active A-tDCS). The mean number of words produced with better accuracy after the intervention in the sham group was $2.17(\mathrm{SD}=1.75)$, while it was $4.42(\mathrm{SD}=3.40)$ for the A-tDCS group (Fig. 2). The difference was statistically significant, after correcting for multiple comparisons $(V=43, p=0.01693)$. The value $V=43$ corresponds to the sum of ranks assigned to the differences with positive sign. Cohen's kappa test was used to compare the agreement rate between the two referees that judged the data. There was no statistical difference between them (anodal: $p=0.5941$, placebo: $p=0.7918$ ).

\section{Qualitative Assessment: Individual Outcomes}

Of the 12 participants, five showed qualitative improvement at follow-up assessments. One 


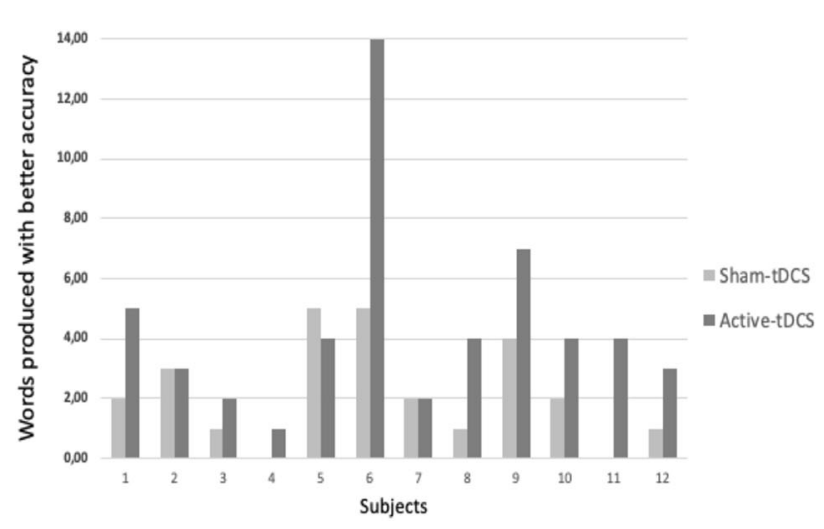

Fig. 2 Articulatory accuracy. Bars represent the difference in word production for each subject before and after the sham tDCS and the active tDCS. Box plot showing the

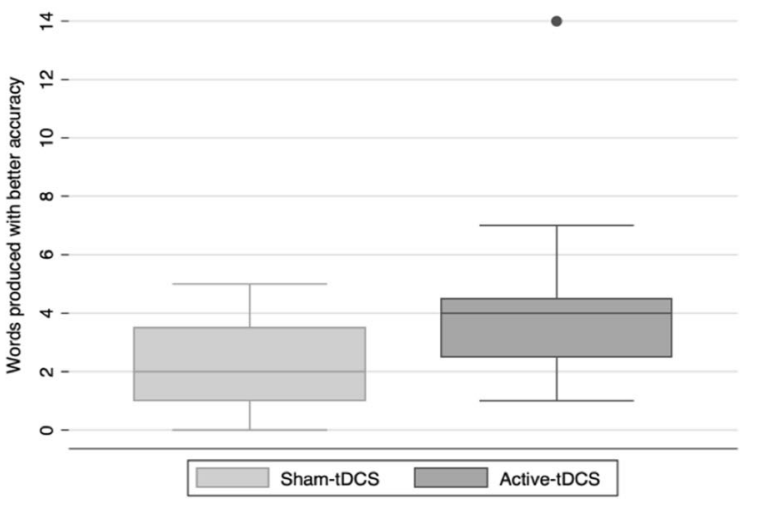

difference between pre- and post-intervention on words produced with better accuracy

Table 3 Results of follow-up assessments for five patients that reported clinical improvement of speech after A-tDCS

\begin{tabular}{lllll}
\hline Subject & $\begin{array}{l}\text { Repetition } \\
\text { deficit }\end{array}$ & $\begin{array}{l}\text { Aphasia classification } \\
\text { before tDCS }\end{array}$ & $\begin{array}{l}\text { Aphasia classification } \\
\text { (follow-up 1) }\end{array}$ & $\begin{array}{l}\text { Aphasia classification } \\
\text { (follow-up 2) }\end{array}$ \\
\hline 3 & Yes & Broca's & Transcortical motor & Transcortical motor \\
4 & Yes & Broca's & Transcortical motor & Transcortical motor \\
6 & Yes & Broca's & Transcortical motor & Transcortical motor \\
7 & No & Anomic & Without aphasia & Without aphasia \\
9 & Yes & Broca's & Transcortical motor & Transcortical motor \\
\hline
\end{tabular}

week after the end of all intervention, at the first follow-up assessment (evaluation 4 in Fig. 1), four subjects had improved repetition ability. The assessments yield to a change in their aphasia's classification. They initially had Broca's aphasia, but recovered into motor transcortical aphasia at follow-up 1 due to significant improvement of word repetition. One subject entirely suppressed the anomia deficit at follow-up 1 and was no longer classified as having aphasia. At a second follow-up assessment 4 months later (evaluation 5 in Fig. 1), these five participants presented the same results (Table 3 ). The same five subjects described above showed improvement on social communication as perceived by themselves, their families, and therapists (Fig. 3). Patient 6, for instance, reported great improvement in spontaneous speech and expressed desire to have more A-tDCS sessions: "quero fazer mais vezes" ("I want to have more"). The same baseline assessments were used in both follow-up sessions.

According to self-perception, eight of 12 participants experienced improvement after A-tDCS or sham. Six family members perceived improvement in the speech of their related participant after A-tDCS or sham. For SLPs, improvement was reported for five participants after A-tDCS or sham and for three participants after A-tDCS only. Agreement among these three groups of raters was low (kappa $=0.105$ ) (Fig. 3). None of the participants, family members, and SLPs reported speech deterioration after either sham or A-tDCS sessions.

\section{DISCUSSION}

In this study, the effect of A-tDCS was tested on: articulatory accuracy, syllable repetition, and 


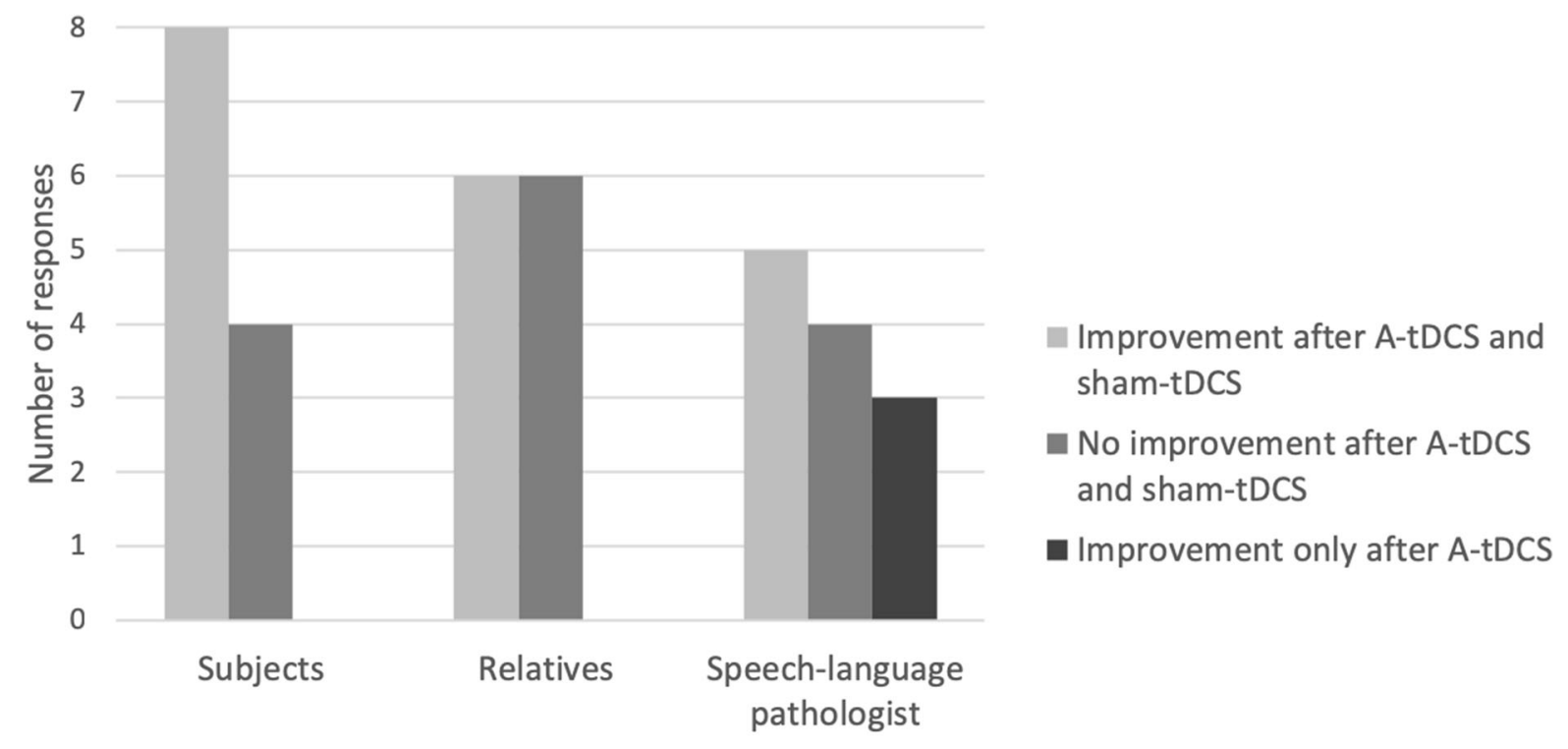

Kappa: $.105 \mathrm{p}=.159$ (Cl 95\% $=-.041$ to .251$)$

Fig. 3 Participants, family, and therapists perceived improvement after A-tDCS and sham sessions. Judgment of participant's speech quality after study interventions by three groups (participant, participant's relatives, and participant's speech-language pathologists). Responses were

word production in patients with aphasia after stroke.

\section{Articulatory Accuracy}

It was observed that articulatory accuracy improved after A-tDCS. The data suggest that ten sessions of $1 \mathrm{~mA}$ A-tDCS over Broca's area can improve motor patterns related to language, as measured by the accuracy assessment.

The results of the potential improvement in articulatory accuracy were evaluated since many studies already suggest the effectiveness of A-tDCS in the improvement of naming ability $[6,12-16,18,20]$. To date, we only found three studies assessing articulatory accuracy after tDCS $[22,39,40]$. These three experiments corroborate our findings on articulatory accuracy improvement influenced by tDCS over Broca's area.

First, Marangolo et al. demonstrated that A-tDCS over Broca's area improved the articulatory ability of subjects with both aphasia and categorized as "improvement", "no improvement", and "worsened". The $Y$-axis represents number of individuals in each group ( $N=12$ in each group) that chose a given category

apraxia [39]. They hypothesized that A-tDCS elicits a prolonged increment in cortical plasticity, probably because of changes in synaptic connections of N-methyl-D-aspartate (NMDA) receptors. Also, stimulation over the left inferior frontal gyrus (IFG) determines long-term effects in the recovery of speech apraxia in patients with chronic aphasia. Second, Marangolo et al. evaluated the number of correct syllables and words produced by subjects with brain damage after bilateral tDCS-A-tDCS over Broca's area and C-tDCS on a homologue area in the right hemisphere [40]. They concluded that bilateral stimulation restored interhemispheric balance, promoting the best outcomes for language recovery. The authors suggested that the improvement in articulatory accuracy was based on the notion that up-regulating the excitability of integral portions of the lesioned hemisphere and down-regulating the excitability of the contralesional hemisphere would lead to the greatest recovery of language.

Finally, Fiori et al. concluded that stimulustDCS over Broca's area improves articulatory 
accuracy in healthy subjects [22]. They suggested that the frontal area could co-activate other premotor areas, leading to improvement in repetition ability and other language tasks. The connectivity of Broca's area with other cortical regions offers the neuroanatomical substrate to explain this effect. The authors also observed a long-term effect of A-tDCS due to a hypothesized lasting depolarization of neurons in the Broca's area.

\section{Syllable Repetition and Word Production}

Performance did not change in the word production and syllable repetition tasks after A-tDCS ( $p=0.15$ and $p=0.79$, respectively) (see Table 2). Although contrary to many studies $[12-16,20]$, this study is not the first to find negative results for the effect of tDCS on naming function. Polanowska et al. applied A-tDCS in 37 subjects with aphasia and did not observe improvement of naming function after tDCS [41]. The same research group in another study with 24 subjects with aphasia also used A-tDCS over Broca's area and found weak evidence for A-tDCS-related language improvement [42]. The systematic review and meta-analysis conducted by Elsner et al. evaluated tDCS against sham tDCS in 12 studies and found no evidence to support the efficacy of intervention as a therapeutic tool for aphasia [6].

Participants' responses differ due to variations in the mechanism of brain plasticity involved in language recovery, which helps in interpreting negative results. Broca's area, located at the frontal lobe and involved in naming processing, was stimulated. However, the temporal area is also linked to naming capability [43]. Location and size of the brain injury are other factors that could explain the lack of effect in our sample [44]. Electrode placement was the same for all individuals (Broca's area), which did not necessarily match the location and extension of each patient's lesion. Moreover, Fridriksson et al. recently found an interaction between a specific genotype of the $B D N F$ gene and the tDCS polarity for treatment-related naming improvement. According to the authors, subjects that carry the genotype val/val
BDNF are more prone to benefit from A-tDCS [45].

Placement of the active electrode is an additional source of mixed results. For instance, Shah-Basak et al., studying seven adults with chronic aphasia, observed improvement on naming tests for three subjects that received anodal stimulation on the left hemisphere, while three others responded positively to cathodal stimulation on the left hemisphere and one subject improved after cathodal stimulation on the right hemisphere [44]. The authors defend individualized use of tDCS electrode montages for stimulation because of individual differences.

Recent evidence suggests that A-tDCS when performed simultaneously with behavioral therapy can improve aphasia-related outcomes $[46,47]$. The offline methodology (stimulation without any other intervention simultaneously) adopted in this study may justify the absence of significant results in word production and syllable repetition.

Several studies suggest that the mechanisms of brain reorganization in patients with lesions caused by a stroke remain largely unknown [48-50], limiting the generalization of tDCS protocols, as a recent meta-analysis showed [6]. More studies are necessary to explore different tDCS electrode montages (size, position, current type) and current intensity (1-2 mA), as well as the use of neuroimaging techniques and transcranial magnetic stimulation (TMS) to help choose the best parameters to treat patients with aphasia. Some authors have begun using functional magnetic resonance imaging (fMRI) concomitantly with tDCS to define the best area to be stimulated [43].

\section{Clinical Improvement}

The current study included a simple qualitative assessment of functional changes after the intervention. While lack of agreement among participants, relatives, and therapists on the effect of t-DCS sessions on participants was observed, it was identified that nearly $70 \%$ of participants $(N=8)$ perceived improvement in their speech production. The higher perception 
among participants in comparison with the other two groups may represent a placebo effect. Nevertheless, subjective perception indirectly measures subject's satisfaction with treatment, which represents a valuable clinical assessment.

Studies looking at the effect of tDCS in the language of subjects with aphasia use several secondary outcomes, complicating a holistic data analysis. Choosing parameters to represent functional improvement is a great challenge. The most common outcomes (e.g., naming accuracy and naming speed) measure isolated word production. Motor planning and organizing during the dialogic context are complex tasks that cannot be fully represented by the usual surrogate outcomes measured in single word production. Interpretation of these outcomes in combination with subjective perception of patients and people surrounding them may give a better understanding of clinical language improvement.

Qualitative improvement was observed even in patients with longer disease onset (patients 3 , 4 , and 9), when not much improvement would normally occur. A recent study including patients with post-stroke chronic aphasia also found that a more severe baseline language profile was associated with larger improvements in one aspect of speech production (fluency) after tDCS [51].

Language is a complex process involving different neural circuits and includes abilities beyond verbal production and comprehension. Little has been investigated about tDCS effects on suprasegmental aspects of language, such as intonation and pitch during speech production [6]. Future tDCS trials should consider investigating these subtle aspects of language both quantitatively and qualitatively.

\section{Limitations}

The current study has limitations that need attention. We had a small sample size, which may have caused a type II error, preventing our ability to find differences between active and sham stimulation for the word production and syllable repetition tasks. A post hoc power analysis, considering the mean and the standard deviation of word production and syllable repetition, indicated we achieved small power for both outcomes ( 0.1027 for word production and 0.1025 for syllable repetition, respectively). A sample size of 95 subjects per group would be necessary to provide power of 0.80 . Nonetheless, other tDCS clinical trials have included a small number of subjects as well, given the difficulties in recruiting and retaining participants with a similar design $[12,13,17,22]$. The crossover design was used to reduce this limitation. Placement of electrodes was theoretically determined for all subjects, as already mentioned above. Ideally, this choice should be determined by neuroimaging assessment to better suit each patient's needs. The lack of effect in this study may have occurred due to placement of the anodal electrode over mostly lesioned brain tissue or necrotic cavities.

We planned to analyze whether there was a carryover effect by comparing the baseline values before active and sham interventions for the group that received active intervention first in the crossover design. However, because of the randomization process, the group that received tDCS treatment first had only two subjects, while the group that received sham treatment first had 10 subjects. This large difference between group sizes hindered our ability to compare the groups regarding the carryover effect of tDCS. Finally, the absence of neuroimaging assessment was an important limitation in this study. This information would have allowed for exploratory analysis regarding location and size of the lesion. We had planned to request these results, but participants came from different healthcare clinics, and most did not have copies of these exams.

\section{CONCLUSIONS}

The current study found differences between pre- and post-A-tDCS stimulation for articulatory accuracy, but not for the number of words produced and number of syllables repeated correctly. The tDCS technique may be a useful therapeutic tool when combined with language, drug, or other therapies. In fact, most studies 
report that brain stimulation promotes improvement of speech and language deficits when combined with specific treatment approaches [26]. We used offline tDCS, but all participants continued receiving standard speech-language therapy outside the study. Our results, along with previous studies that explored the use of tDCS as a treatment tool to help improve speech ability, are encouraging in this search for more effective ways to treat patients with aphasia.

The evidence presented here is not sufficient to establish tDCS as an effective therapeutic tool in the rehabilitation of subjects with aphasia. Further studies with larger sample sizes and different montage combinations are needed. However, in the absence of larger studies, we suggest the use of multiple therapeutic methods, including trials with tDCS in research settings, as an effective approach to benefit these patients.

\section{ACKNOWLEDGEMENTS}

We are grateful to this trial's participants. We thank Zeus Moreira and Obras Sociais Irmã Dulce for the support to conduct this study at the Santo Antônio Hospital (Salvador-BA, Brazil). Thanks to Antônio Mattos, Carla Filgueiras, Lorena Argolo, Lorene Lins, Henrique Porto, Lais Bittencourt, and Liliane Medeiros for assistance with data collection. We are also thankful to professors Paulo Sergio Boggio (Universidade Presbiteriana Mackenzie) and Felipe Fregni (Harvard Medical School) for their suggestions throughout the development of this study.

Funding. No funding or sponsorship was received for this study or publication of this article.

Authorship. All named authors meet the International Committee of Medical Journal Editors (ICMJE) criteria for authorship for this article, take responsibility for the integrity of the work as a whole, and have given their approval for this version to be published.
Disclosures. Camila Vila-Nova received a doctoral scholarship from the Brazilian National Council for Scientific and Technological Development $(\mathrm{CNPq})$ during the time of data collection. Fernanda Campbell received a post-doctoral scholarship from the Coordination for the Improvement of Higher Education Personnel (CAPES) during manuscript writing. Pedro H. Lucena, Rita Lucena, and Giulia Armani-Franceschi have nothing to disclose.

Compliance with Ethics Guidelines. All procedures performed in studies involving human participants were in accordance with the ethical standards of the institutional and/or national research committee and with the 1964 Declaration of Helsinki and its later amendments or comparable ethical standards. Informed consent was obtained from all individual participants included in the study. The study was approved by the Hospital Santo Antonio's IRB, Salvador-Bahia, Brazil (\#22/07).

Data Availability. The datasets generated during and/or analyzed during the current study are available from the corresponding author on reasonable request.

Open Access. This article is distributed under the terms of the Creative Commons Attribution-NonCommercial 4.0 International License (http://creativecommons.org/licenses/ by-nc/4.0/), which permits any noncommercial use, distribution, and reproduction in any medium, provided you give appropriate credit to the original author(s) and the source, provide a link to the Creative Commons license, and indicate if changes were made.

\section{REFERENCES}

1. Engelter ST, Gostynski M, Papa S, Frei M, Born C, Ajdacic-Gross $V$, et al. Epidemiology of aphasia attributable to first ischemic stroke: Incidence, severity, fluency, etiology, and thrombolysis. Stroke. 2006;37(6):1379-84.

2. Glize B, Villain M, Richert L, Vellay M, De Gabory I. Language features in the acute phase of poststroke 
severe aphasia could predict the outcome. Eur J Phys Rehabil Med. 2017;53(2):249-55.

3. Dhamoon M, Moon Y, Paik M, Sacco R, Elkind M. Trajectory of Functional Decline Before and After Ischemic Stroke. Stroke. 2012;43(8):2180-4.

4. Meister IG, Boroojerdi B, Foltys H, Sparing R, Huber W, Töpper R. Motor cortex hand area and speech: implications for the development of language. Neuropsychologia. 2003;41(4):401-6.

5. Bose A, van Lieshout P. Effects of utterance length on lip kinematics in aphasia. Brain Lang. 2008;106(1):4-14.

6. Elsner B, Kugler J, Pohl M, Mehrholz J. Transcranial direct current stimulation (tDCS) for improving aphasia in patients with aphasia after stroke. Cochrane Database Syst Rev. 2015;1(5):CD009760.

7. Sebastian R, Tsapkini K, Tippett DC. Transcranial direct current stimulation in post stroke aphasia and primary progressive aphasia: Current knowledge and future clinical applications. NeuroRehabilitation. 2017;39(1):141-52.

8. Cohen Kadosh R, Soskic S, Iuculano T, Kanai R, Walsh V. Modulating neuronal activity produces specific and long-lasting changes in numerical competence. Curr Biol. 2010;20(22):2016-20.

9. Flöel A. tDCS-enhanced motor and cognitive function in neurological diseases. Neuroimage. 2014;85(Part 3):934-47.

10. Fiebach CJ, Schlesewsky M, Lohmann G, von Cramon DY. Revisiting the role of Broca's area in sentence processing: syntactic integration versus syntactic working memory. Hum Brain Mapp. 2005;24(2):79-91.

11. Lesser RP, Lueders H, Dinner DS, Hahn J, Cohen L. The location of speech and writing functions in the frontal language area. Results of extraoperative cortical stimulation. Brain (London, Engl 1878). 1984;107(1):275; 275-291; 291.

12. Baker JM, Rorden C, Fridriksson J. Using transcranial direct-current stimulation to treat stroke patients with aphasia. Stroke. 2010;41(6):1229-36.

13. Fridriksson J, Richardson JD, Baker JM, Rorden C. Transcranial direct current stimulation improves naming reaction time in fluent aphasia: a doubleblind, sham-controlled study. Stroke. 2010;42(3):819-21.

14. Jung IY, Lim JY, Kang EK, Sohn HM, Paik NJ. The factors associated with good responses to speech therapy combined with transcranial direct current stimulation in post-stroke aphasic patients. Ann Rehabil Med. 2011;35(4):460-9.

15. de Aguiar V, Paolazzi CL, Miceli G. tDCS in poststroke aphasia: the role of stimulation parameters, behavioral treatment and patient characteristics. Cortex [Internet]. 2015;63:296-316. https://doi. org/10.1016/j.cortex.2014.08.015.

16. Marangolo P, Fiori V, Di Paula M, Cipollari S, Razzano C, Oliveri M, et al. Differential involvement of the left frontal and temporal regions in verb naming: a tDCS treatment study. Restor Neurol Neurosci. 2013;31(1):63-72.

17. Lee SY, Cheon H-J, Yoon KJ, Chang WH, Kim Y-H. Effects of dual transcranial direct current stimulation for aphasia in chronic stroke patients. Ann Rehabil Med. 2013;37(5):603-10.

18. Monti A, Cogiamanian F, Marceglia S, Ferrucci R, Mameli F, Mrakic-Sposta S, et al. Improved naming after transcranial direct current stimulation in aphasia. J Neurol Neurosurg Psychiatry. 2008;79(4):451-3.

19. Vines BW, Norton AC, Schlaug G. Non-invasive brain stimulation enhances the effects of melodic intonation therapy. Front Psychol. 2011;2:230.

20. Flöel A, Meinzer M, Kirstein R, Nijhof S, Deppe M, Knecht $S$, et al. Short-term anomia training and electrical brain stimulation. Stroke. 2011;42(7):2065-7.

21. Iyer MB, Mattu U, Grafman J, Lomarev M, Sato S, Wassermann EM. Safety and cognitive effect of frontal DC brain polarization in healthy individuals. Neurology. 2005;64(5):872-5.

22. Fiori V, Cipollari S, Di Paola M, Razzano C, Caltagirone C, Marangolo P. tDCS stimulation segregates words in the brain: evidence from aphasia. Front Hum Neurosci. 2013;14(7):269.

23. Goldstein LB, Jones MR, Matchar DB, Edwards LJ. Improving the reliability of stroke subgroup classification using the Trial of ORG 10172 in Acute Stroke Treatment (TOAST) criteria. Stroke (1970). 2001;32(5):1091-6.

24. Jakubovicz R. Teste de Reabilitação das Afasias. Rio de Janeiro: Livraria e Editora Revinter; 2005.

25. Boggio PS, Campanhã C, Valasek CA, Fecteau S, Pascual-Leone A, Fregni F. Modulation of decisionmaking in a gambling task in older adults with transcranial direct current stimulation. Eur J Neurosci. 2010;31(3):593-7.

26. Marangolo P, Fiori V, Calpagnano MA, Campana S, Razzano C, Caltagirone C, et al. tDCS over the left 
inferior frontal cortex improves speech production in aphasia. Front Hum Neurosci. 2013;7:539.

27. DaSilva AF, Volz MS, Bikson M, Fregni F. Electrode positioning and montage in transcranial direct current stimulation. J Vis Exp. 2011;51(e2744):1-12.

28. Cattaneo Z, Pisoni A, Papagno C. Transcranial direct current stimulation over Broca's region improves phonemic and semantic fluency in healthy individuals. Neuroscience. 2011;183:64-70.

29. Hesse S, Werner C, Schonhardt EM, Bardeleben A, Jenrich W, Kirker SG. Combined transcranial direct current stimulation and robot-assisted arm training in subacute stroke patients: a pilot study. Restor Neurol Neurosci. 2007;25(1):9-15.

30. Nitsche MA, Fricke K, Henschke U, Schlitterlau A, Liebetanz D, Lang N, et al. Pharmacological modulation of cortical excitability shifts induced by transcranial direct current stimulation in humans. J Physiol. 2003;553(Pt 1):293-301.

31. Thair H, Holloway AL, Newport R, Smith AD. Transcranial direct current stimulation (tDCS): a beginner's guide for design and implementation. Front Neurosci [Internet]. 2017;11:641.

32. Pompéia S, Miranda MC, Bueno OFA. Brazilian standardised norms for a set of pictures are comparable with those obtained internationally. Arq Neuropsiquiatr. 2003;61(4):916-9.

33. Snodgrass J, Vanderwart M. A standardized set of 260 pictures: norms for name agreement, image agreement, familiarity, and visual complexity. J Exp Psychol Hum Learn. 1980;6(2):174-215.

34. Fadiga L, Craighero L, D'Ausilio A. Broca's area in language, action, and music. Ann N Y Acad Sci. 2009; 1169:448-58.

35. Goodwin C. Conversational framework of accomplishment of meaning in aphasia. In: Goodwin $\mathrm{C}$ (ed). Conversation and brain damage. 1st edn. New York: Oxford University Press; 2002. p. 90-116.

36. Lesser R, Milroy L. Linguistics and aphasia: psycholinguistic and pragmatic aspects of intervention. New York: Taylor \& Frances; 2003.

37. Landis JR, Koch GG. The measurement of observer agreement for categorical data. Biometrics. 1977;33(1):159-74.

38. RDC T. R: A language and environment for statistical computing. Vol. 2010. 2010.

39. Marangolo P, Marinelli CV, Bonifazi S, Fiori V, Ceravolo MG, Provinciali L, et al. Electrical stimulation over the left inferior frontal gyrus (IFG) determines long-term effects in the recovery of speech apraxia in three chronic aphasics. Behav Brain Res. 2011;225(2):498-504.

40. Marangolo P, Fiori V, Sabatini U, De Pasquale G, Razzano C, Caltagirone C, et al. Bilateral transcranial direct current stimulation language treatment enhances functional connectivity in the left hemisphere: preliminary data from aphasia. J Cogn Neurosci. 2016;28(5):724-38.

41. Polanowska KE, Lesniak M, Seniow JB, Czlonkowska A. No effects of anodal transcranial direct stimulation on language abilities in early rehabilitation of post-stroke aphasic patients. Neurol Neurochir Pol. 2013;47(5):414-22.

42. Polanowska KE, Lesniak MM, Seniow JB, Czepiel W, Czlonkowska A. Anodal transcranial direct current stimulation in early rehabilitation of patients with post-stroke non-fluent aphasia: a randomized, double-blind, sham-controlled pilot study. Restor Neurol Neurosci. 2013;31(6):761-71.

43. Holland R, Leff AP, Josephs O, Galea JM, Desikan M, Price CJ, et al. Speech facilitation by left inferior frontal cortex stimulation. Curr Biol. 2011;21(16):1403-7.

44. Shah-Basak P, Norise C, Garcia G, Torres J, Faseyitan $\mathrm{O}$, Hamilton RH. Individualized treatment with transcranial direct current stimulation in patients with chronic non-fluent aphasia due to stroke. Front Hum Neurosci. 2015;9:201.

45. Fridriksson J, Elm J, Stark BC, Basilakos A, Rorden C, Sen $S$, et al. BDNF genotype and tDCS interaction in aphasia treatment. Brain Stimul. 2018;11(6):1276-81.

46. Fridriksson J, Basilakos A, Stark BC, Rorden C, Elm J, Gottfried $M$, et al. Transcranial direct current stimulation to treat aphasia: longitudinal analysis of a randomized controlled trial. Brain Stimul Basic Transl Clin Res Neuromodul [Internet]. 2019;12(1):190-1. https://doi.org/10.1016/j.brs. 2018.09.016.

47. Fridriksson J, Rorden C, Elm J, Sen S, George MS, Bonilha L. Transcranial direct current stimulation vs sham stimulation to treat aphasia after stroke: a randomized clinical trial. JAMA Neurol. 2018;75(12):1470-6.

48. Winhuisen L, Thiel A, Schumacher B, Kessler J, Rudolf J, Haupt WF, et al. The right inferior frontal gyrus and poststroke aphasia: a follow-up investigation. Stroke. 2007;38(4):1286-92.

49. Gold BT, Buckner RL. Common prefrontal regions coactivate with dissociable posterior regions during 
controlled semantic and phonological tasks. Neuron. 2002;35(4):803-12.

50. Cappa SF, Benke T, Clarke S, Rossi B, Stemmer B, van Heugten CM, et al. EFNS guidelines on cognitive rehabilitation: report of an EFNS task force. Eur J Neurol. 2005;12(9):665-80.
51. Norise C, Sacchetti D, Hamilton R. Transcranial direct current stimulation in post-stroke chronic aphasia: the impact of baseline severity and task specificity in a pilot sample. Front Hum Neurosci [Internet]. 2017;11(May):1-12. https://doi.org/10. 3389/fnhum.2017.00260/full. 